\title{
Biallelic PDE2A variants: a new cause of syndromic paroxysmal dyskinesia
}

\author{
Diane Doummar ${ }^{1,2} \cdot$ Christel Dentel $^{3} \cdot$ Romane Lyautey $^{4} \cdot$ Julia Metreau $^{5} \cdot$ Boris Keren $^{6} \cdot$ Nathalie Drouot $^{7,8}$. \\ Ludivine Malherbe $^{4}$. Viviane Bouilleret ${ }^{9} \cdot$ Jérémie Courraud $^{7,8} \cdot$ Maria Paola Valenti-Hirsch $^{10} \cdot$ Lorella Minotti $^{11}$. \\ Blandine Dozieres-Puyravel ${ }^{12}$. Séverine Bär ${ }^{4}$. Julia Scholly ${ }^{10}$. Elise Schaefer ${ }^{13,14}$. Caroline Nava ${ }^{6}$. \\ Thomas Wirth $\mathbb{D}^{15}$. Hala Nasser ${ }^{16,17} \cdot$ Marie de Salins ${ }^{1} \cdot$ Anne de Saint Martin ${ }^{18}$ - Marie Thérèse Abi Warde ${ }^{18}$. \\ Philippe Kahane $^{11} \cdot$ Edouard Hirsch $^{10} \cdot$ Mathieu Anheim ${ }^{7,8,19} \cdot$ Sylvie Friant $\mathbb{B}^{4} \cdot$ Jamel Chelly $y^{7,8,20}$. \\ Cyril Mignot ${ }^{6,21,22} \cdot$ Gabrielle Rudolf $\mathbb{C}^{7,8,10,15}$
}

Received: 31 May 2019 / Revised: 1 April 2020 / Accepted: 28 April 2020 / Published online: 28 May 2020

(c) The Author(s), under exclusive licence to European Society of Human Genetics 2020

\begin{abstract}
Cause of complex dyskinesia remains elusive in some patients. A homozygous missense variant leading to drastic decrease of PDE2A enzymatic activity was reported in one patient with childhood-onset choreodystonia preceded by paroxysmal dyskinesia and associated with cognitive impairment and interictal EEG abnormalities. Here, we report three new cases with biallelic PDE2A variants identified by trio whole-exome sequencing. Mitochondria network was analyzed after Mitotracker ${ }^{\mathrm{TM}}$ Red staining in control and mutated primary fibroblasts. Analysis of retrospective video of patients' movement disorder and refinement of phenotype was carried out. We identified a homozygous gain of stop codon variant c.1180C $>$ T; p. (Gln394*) in PDE2A in siblings and compound heterozygous variants in young adult: a missense c.446C $>\mathrm{T}$; p.(Pro149Leu) and splice-site variant c.1922+5G $>$ A predicted and shown to produce an out of frame transcript lacking exon 22. All three patients had cognitive impairment or developmental delay. The phenotype of the two oldest patients, aged 9 and 26, was characterized by childhood-onset refractory paroxysmal dyskinesia initially misdiagnosed as epilepsy due to interictal EEG abnormalities. The youngest patient showed a proven epilepsy at the age of 4 months and no paroxysmal dyskinesia at 15 months. Interestingly, analysis of the fibroblasts with the biallelic variants in PDE2A variants revealed mitochondria network morphology changes. Together with previously reported case, our three patients confirm that biallelic PDE2A variants are a cause of childhood-onset refractory paroxysmal dyskinesia with cognitive impairment, sometimes associated with choreodystonia and interictal baseline EEG abnormalities or epilepsy.
\end{abstract}

These authors contributed equally: Diane Doummar, Christel Dentel, Romane Lyautey

These authors jointly supervised this work: Cyril Mignot, Gabrielle Rudolf

Supplementary information The online version of this article (https:// doi.org/10.1038/s41431-020-0641-9) contains supplementary material, which is available to authorized users.

Gabrielle Rudolf

Gabrielle.Rudolf@chru-strasbourg.fr

Extended author information available on the last page of the article

\section{Introduction}

Paroxysmal dyskinesias (PD) are a heterogeneous group of rare hyperkinetic movement disorders characterized by recurrent and sudden attacks of dystonic and/or choreic involuntary movements [1-3]. Several genes have been associated with different types of PD with normal interictal examination: PRRT2 is the main gene for paroxysmal kinesigenic dyskinesia (PKD) sometimes with epilepsy [4]; paroxysmal exercise-induced dyskinesia (PED) is mainly related to some SLC2Al variants [5]; paroxysmal nonkinesigenic dyskinesia (PNKD) is related to alterations in $M R 1$ [6]. More complex phenotypes associating PD and chronic interictal chorea or choreodystonic movement disorder and/or epilepsy may be due to $S L C 2 A 1$ or $A D C Y 5$ variants [7]. None of these genes were identified as disease 
causing in our patients. $P D E 2 A$ seems to be a new candidate gene for syndromic paroxysmal dyskinesia.

More recently, Salpietro et al. reported a missense homozygous variant in $P D E 2 A$ in a patient with cognitive impairment, interictal EEG abnormalities, and childhoodonset chorea [8]. Disease onset consisted in fluctuating attacks of sudden falls, followed by dystonic postures and generalized choreic movements suggesting paroxysmal dyskinesia. Here we report on three new patients (two siblings and a sporadic case), including two with PD and one with epilepsy, who underwent whole-exome sequencing (WES) revealing biallelic $P D E 2 A$ variants.

In addition, a $P D E 2 A$ isoform was reported to be localized in mitochondria and regulates cellular energy metabolism [9]. Mitochondria is a dynamic network that is continuously remodeled by fusion and fission reactions. Imbalanced fusion/fission reactions contribute to the pathogenesis of complex conditions. Mitochondrial dysfunction in brain, an intensely energy-dependent tissue, could play a role in the pathophysiology of hyperkinetic movement disorder associated at disease onset with cortical hyperexcitability. Here we report that abnormal mitochondria network is present in patient\#3 and \#1 with biallelic $P D E 2 A$ variants.

\section{Materials and methods}

All procedures were carried out with the adequate understanding and written consent of the patient and her both parents, and according to the appropriate ethical committee ( ${ }^{\circ}$ CPP 09/40-DC-2009-1002/DC-2014-2222). Consent for videos was obtained from the proband and their parents.

\section{Whole-exome sequencing}

DNA was extracted using standard procedures and trio WES was performed on a HiSeq 1000 Sequencing System (Illumina, San Diego, CA), with a $2 \times 150$ bp high output sequencing kit after enrichment with SeqCap EZ MedExome kit (Roche, Basel, Switzerland), according to manufacturer's specifications. Sequence quality was assessed with FastQC 0.11.5, then the reads were mapped using BWA-MEM (version 0.7.13), sorted and indexed in a bam file (Samtools 1.4.1), duplicates were flagged (Sambamba 0.6.6), coverage was calculated (Picard-Tools 2.10.10). Variant calling was done with GATK 3.7 Haplotype Caller. Variants were then annotated with SnpEff 4.3, dbNSFP 2.9.3, gnomAD, ClinVar, HGMD, Variome Great Middle East and an internal database. Coverage for these patients was $93 \%$ at a $20 \times$ depth threshold.

\section{Sanger sequencing}

After exome analysis, $P D E 2 A$ variants were confirmed by Sanger sequencing using patient's, and parents' DNA. Sequencing of PCR amplified fragments was performed on an ABI 3100 with the Big Dye Sequencing Kit according to the manufacturer's specifications (Thermo Fischer Scientific, Waltham, MA).

\section{RT-PCR}

In order to investigate the consequences of the c.1922 $+5 \mathrm{G}>\mathrm{A}$ variant located in the vicinity of exon 22 , total RNA was purified from blood sample collected on PAXgene (Qiagen) and fibroblasts, then cDNA was synthesized using the SuperScript II Reverse Transcriptase kit (Invitrogen). Polymerase Chain Reaction (PCR) was used to amplify the cDNA using a first couple of primers located in exons 21 and 24. Nested RT-PCR was performed using the initial PCR reaction product as template for the second round of amplification using a set of primers internal to the one used in the first. To specifically amplify and sequence the predicted transcript lacking exon 22 resulting from the c. $1922+5 \mathrm{G}>\mathrm{A}$ variant, we used, for the nested PCR, a forward primer that covers both the $3^{\prime}$ end of exon 21 and the $5^{\prime}$ end of exon 23. PCR products were run on a $1 \%$ agarose gel and Sanger-sequenced using Big Dye Sequencing Kit (Thermo Fischer Scientific, Waltham, MA) and ABI 3100 equipment according to the manufacturer's specifications.

\section{In silico splice site prediction}

To evaluate the potential pathogenicity impact of the $\mathrm{C}>\mathrm{T}$ of the splice site variation on the efficiency of splicing, in silico analysis was performed using prediction bioinformatics tools available in Alamut ${ }^{\circ}$ Visual interactive biosoftware.

\section{Mitochondria morphology analysis}

Primary skin fibroblasts from patient\#1 and \#3 and a control individual were grown in DMEM supplemented with $10 \%$ fetal calf serum and $1 \%$ penicillin-streptomycin-glutamin at $37^{\circ} \mathrm{C}$ with $5 \% \mathrm{CO}_{2}$. Cells incubated with $50 \mathrm{nM}$ Mitotracker ${ }^{\mathrm{TM}}$ Red CMXRos (Invitrogen) for $30 \mathrm{~min}$ were fixed with paraformaldehyde $4 \%$ prior to observation on a fluorescence microscope (Zeiss Axio Observer D1) in Vectashield Antifade Mounting Medium bearing DAPI to stain the nucleus [10]. 100 cells were counted for each individual in three independent experiments, and the percentage of cells displaying aberrant mitochondria phenotype 
was determined, the mean was calculated and significance was determined using a $T$ test.

\section{Results}

\section{Clinical cases description and genetic results}

Patient \#l is the third child of healthy related parents from Moroccan origin. Fetal sonography showed intrahepatic portosystemic venous shunt during pregnancy (later successfully treated, at 2 years of age). Delivery occurred at term with a birth weight of $3820 \mathrm{~g}$, length $49 \mathrm{~cm}$, and occipitofrontal circumference (OFC) $36 \mathrm{~cm}$. At the age of 7 months, she started to experience bursts of brief tonic symmetric contractions or extension of her four limbs and upward eye deviation occurring during awakening or when falling asleep (Video1-Segment 1). Psychomotor regression was noticed by her parents. Spasms were considered but EEG recording showed no electrical correlates or hypsarrhythmia. It showed interictal fronto-central spikes with left predominance. Vigabatrin treatment was introduced, then spasms disappeared and Patient \#1 recovered her psychomotor development. She sat at 9 months, could stand with support, acquired pincer grip, and walked a few steps by 24 months. At 17 months, other movement disorders appeared consisting in sudden backward propulsion of the body, opening of the mouth, sometimes with head turning to the left or to the right, extension of four limbs, then brisk and repetitive but nonrhythmic chorea-dystonic movement of all limbs lasting $<1$ min (Video1-Segment 3). These attacks were most of the time spontaneous, could occur during sleep (Video1-Segment 4), and were sometimes triggered by sudden changes of body position or by unexpected auditory stimuli. Several bursts of attacks occurred daily and increased with times so that Patient \#1 became unable to walk. Holter EEG at 4 years old showed interictal asymptomatic bilateral fronto-central theta waves and artifacts during paroxysms; thus, epilepsy could not be ruled out. Hydrocortisone therapy was introduced without benefits. At the age of 8 years, Patient \#1 still had more than 100 attacks per day. At that time, paroxysmal dyskinesia was considered as the most appropriate diagnosis. Trials of carbamazepine, oxcarbamazepine, ketogenic diet, levodopa, acetazolamide, zonisamide, and cyamemazine were also inefficient. At examination, she had normal OFC, marked truncal hypotonia, permanent choreic movements of the limbs, and dystonic postures of hands. She could stand but did not walk unaided. She was a smiling child willing to communicate (Video1-Segment 2): she spoke a few words, used words of sign language and pictograms, and could understand simple orders. She could catch object and carry some food to her mouth but most of the time she needed to be fed by her mother.
Brain MRI of Patient \#1 was normal. Screening for inherited disorders of metabolism, sequencing of the ADCY5 gene, DNA study with the TruSight One gene panel [11] were all normal. Trio-based WES showed the following Sangerverified homozygous variant in the PDE2A gene: chr11 (GRCh37):g.72297116G>A; NC_000011.9 (NM_002599.4; 31 exons numbered consecutively from 1 to 31 ):c.1180C $>\mathrm{T}$; p.(Gln394*), with each parent being heterozygous (Fig. 1). This variant, never reported in public database, was predicted to activate the nonsense-mediated RNA decay resulting in an absent protein with a CADD phred score of 49.

Patient \#2 was the younger brother of Patient \#1. Prenatal sonography showed intrahepatic portosystemic shunt. Delivery was induced at 37 weeks of gestation because of decreased fetal heart rate with birth weight of $3130 \mathrm{~g}$, length $49 \mathrm{~cm}$, and OFC $33 \mathrm{~cm}$. At the second day of life, he had severe bradycardia and episodes of hypoglycemia related to insufficient feeding. He was discharged at 3 weeks of life with normal examination. The portosystemic shunt spontaneously resolved. First movement disorders appeared when the child was 3 months. EEG was performed because of "startles" without electrical correlate and showed interictal sporadic spikes. At 4 months, Patient \#2 had series of brief tonic extensions of both arms with upward eye deviation and mouthing. Ictal EEG recording showed epileptic spasms and right frontal seizures. Interictal EEG showed slow waves and multifocal spikes. Brain MRI was normal. Patient \#2 received vigabatrin promptly associated with prednisolone. Spasms initially responded to this treatment but relapsed one month later requiring initiation of a ketogenic diet. Clinical examination at 4 months showed an interactive infant with truncal hypotonia and normal OFC ( -2 SD). At 5 months, Patient \#2 experienced a focal status epilepticus lasting for $24 \mathrm{~h}$. At 12 months, he could hold his head, had fair interactions but could not sit unaided or catch objects. He had not showed dyskinesia to date. The PDE2A homozygous c.1180C $>$ T; p. $(\mathrm{Gln} 394 *)$ variation was also found in this patient (Fig. 1).

Patient \#3. This 26-year-old male patient is the unique child of healthy unrelated parents. Pregnancy, delivery (occurred at 39 weeks of gestation with a birth weight of $3140 \mathrm{~g}$, length $51 \mathrm{~cm}$ ), and neonatal period were unremarkable. He was referred at 3 years of age because of speech delay which revealed global cognitive impairment. Neurological examination was normal. Paroxysmal involuntary movements started at 7 years of age. They were characterized in the major part of cases by a first sterotyped dystonic/tonic phase lasting for 5-10 s during which he had speech arrest and axial anteflexion of the trunk, head and eye deviation to the left, followed by head and eye rotation to the right, tonic elevation of the left limbs, and inferior facial grimacing. This was immediately followed by a second choreo-athetosic phase lasting for 2-5 min with 

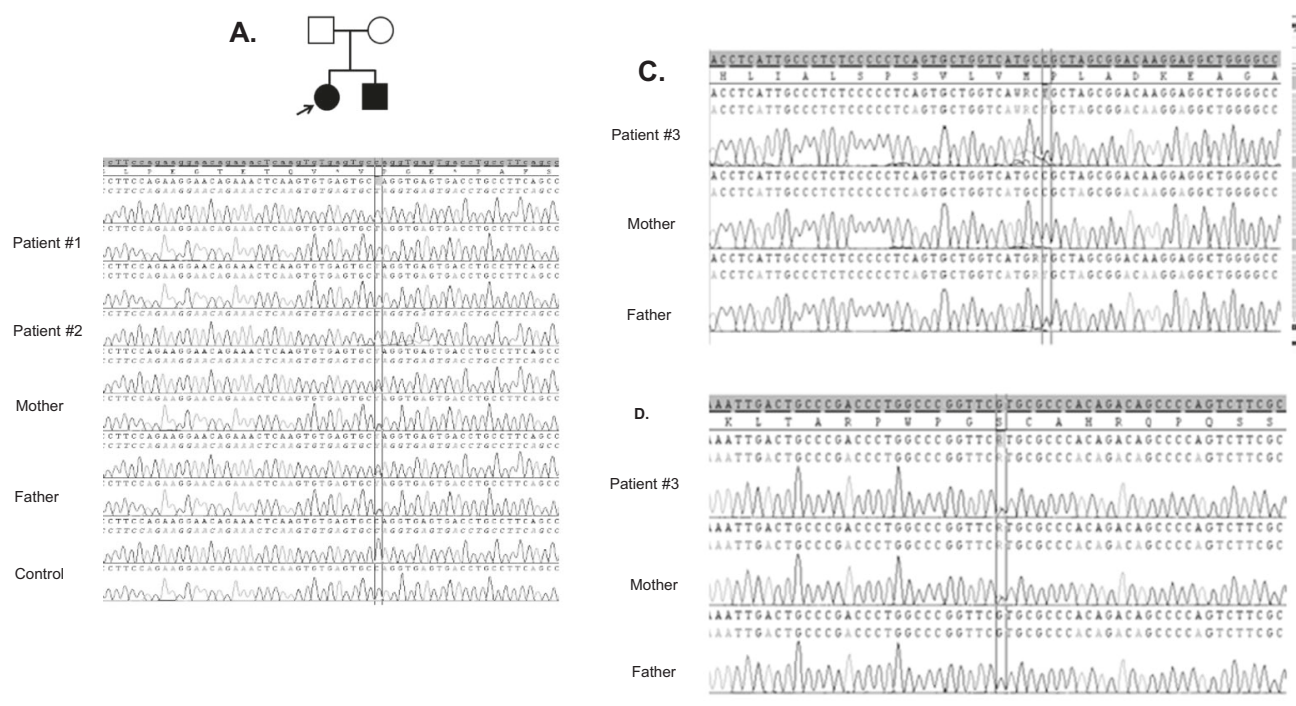

Fig. 1 Pedigree and chromatograms of genomic DNA sequence of biallelic variations in $P D E 2 A$ patients reported in this study. a Pedigree of Patient \# 1 who was confirmed by Sanger sequencing to be homozygous for the c.1180C $>\mathrm{T}$; p.(Gln394*) nonsense variant and both parents being heterozygous. $\mathbf{b}$ Pedigree and Sanger sequencing of Patient \#3 showing heterozygous compound variation. c missense

movements of the four limbs, predominantly on upper limbs, of the trunk, tongue and head, preserved consciousness and dysarthria. Sometimes, dystonic phase occurred alone or dystonic and choreic phases occurred at the same time (Video 2, three first sequences).

Movement disorders worsened with onset of many falls at 13-year-old. Neurological interictal examination was still normal. Neuropsychological evaluation still showed significant intellectual disability (verbal IQ 52, performance IQ 46, full scale IQ 46). Scalp video-EEG monitoring pointed out normal background activity and interictal synchronous rhythmic $10 \mathrm{~Hz}$ polyspikes of $5 \mathrm{~s}$ duration over bilateral anterior frontal region with left predominance and bi-frontal sharp waves but no ictal EEG abnormalities. Brain MRI was unremarkable. Eighteen fluorodesoxy-glucose cerebral TEP-scan evidenced a left posterior frontal hypermetabolism and a bitemporal hypometabolism. Taking into account, stereotyped dystonic/tonic phase, interictal EEG abnormalities, and left frontal hypermetabolism on TEPscan, the hypothesis of focal epilepsy implicating a fronto-mesial network was evoked and a broad spectrum of anticonvulsivant agents (oxcarbamazepine, vigabatrin, acetazolamid, valproic acid, levetiracetam, carbamazepine, clobazam, clonazepam, zonisamide, topiramate, phenytoin, lacosamid, lamotrigin, phenobarbital, eslicarbazepine, rufinamid, and ketogenic diet) was used with little or no
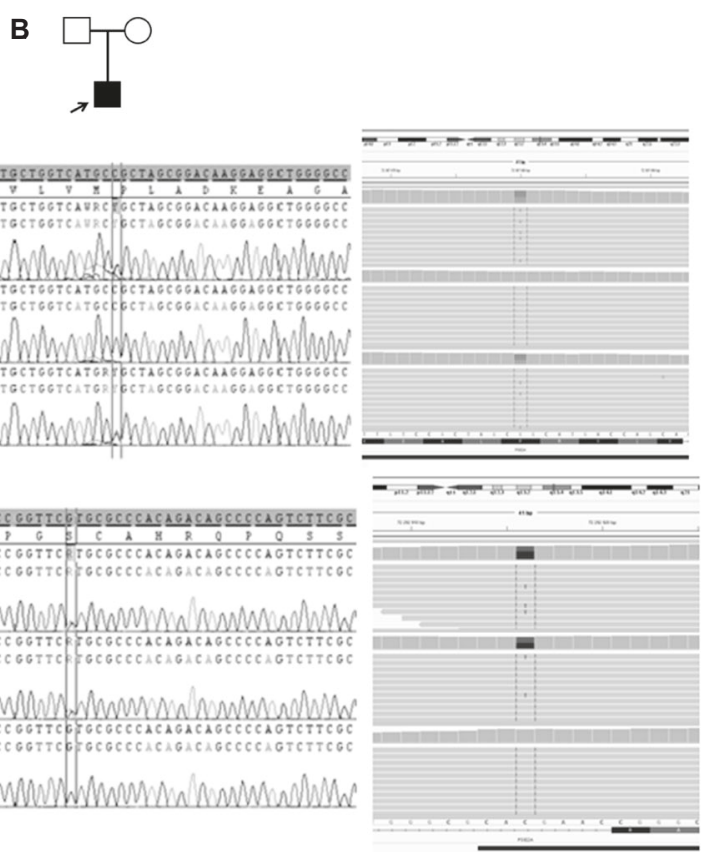

variant c.446C>T; p.(Pro149Leu) herited from the unaffected father and $\mathbf{d}$ splice site variant c. $1922+5 \mathrm{G}>\mathrm{A}$ inherited from the unaffected mother. Reads from exome sequence data are shown with Sanger sequences. Circles represent female participants and squares male participants. Black symbols indicate affected patients. Arrows indicate the proband.

improvement. At that time, Patient \#3 underwent SEEG (Stereo-Electro-Encephalography) with bilateral exploration of the frontal lobe without ictal discharges recording. Thus, reflex dyskinetic paroxystic movements was considered and genetic analyses (PRRT2, MRI, and SLC2Al) were performed with no evidenced variation.

At 26 years, choreic phase seems more attenuate and dystonic phase becomes more tonic and specific. In fact, tonic/dystonic phase begins with a particular facial feature characterized by the turned-down mouth, describe in French as the "chapeau de gendarme sign" [12] followed by a tonic elevation of left limbs and flexion of the right arm (Video 2 fourth sequence). Some of these attacks were preceded by hearing of a helicopter noise in both ears. These attacks occurred 30-50 times per day with a diurnal predominance. They were triggered by stress, sudden auditory, visual and tactile stimuli, but not by sudden movements. Exercise seemed to improve and decrease the attacks.

Trio-based WES identified compound heterozygous variations in $P D E 2 A$, a missense variant chr11: 72307680G >A， NC_000011.9 (NM_002599.4):c.446C>T; p.(Pro149Leu) inherited from his healthy mother and a splice site variant chr11:72292916C $>$ T; NC_000011.9 (NM_002599.4): c.1922+5G $>$ A (predicted to disrupt exon 22 splicing) inherited from his healthy father. Both variants are not reported in public databases (ExAc, Gnomad). 


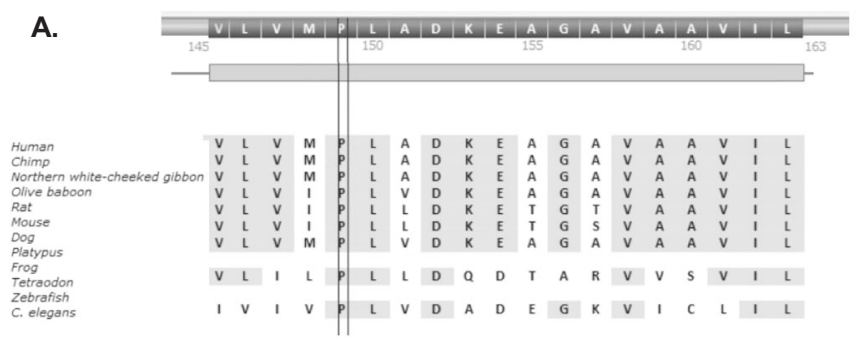

B.

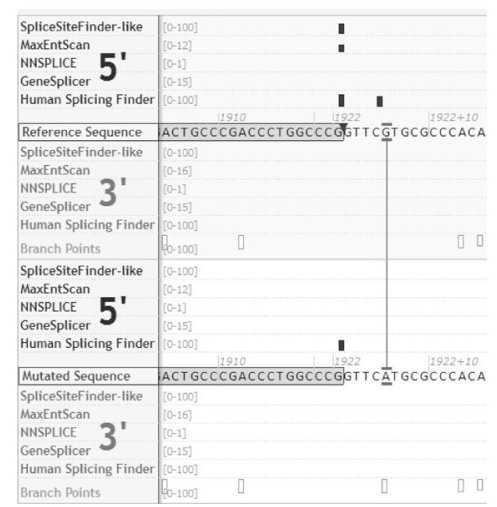

Fig. 2 In silico experimental data for the biallelic PDE2A variants in Patient \#3. a Multiple-sequence alignment showing the highly conserved Pro140 amino acid in the protein sequence encoded by the missense mutation c.446C $>$ T; p.(Pro149Leu). b Bioinformatics analyses predicting a drastic decrease in the efficiency of the canonical donor splice site predicted to lead to exon 22 skipping. c Schematic representation of RT-PCR (primer sequence used for RT-PCR: EX21F1:5'CCACATGAAGGTCTCCGATG3'-EX23R1:5'GAGTTG

These WES findings were confirmed by Sanger sequencing (Fig. 1). The missense variant p.(Pro149Leu) affects a highly conserved amino acid of the protein sequence across species (Fig. 2a). It is located upstream of both regulatory GAF-A and GAF-B domains and was predicted to be disease causing (Mutation Taster). Moreover, PDE2A gene was found to be highly constrained for missense variant $(z=4.78)$ [13]. For the $\mathrm{c} .1922+5 \mathrm{G}>\mathrm{A}$ variant, bioinformatics analyses predicted a drastic decrease in the efficiency of the canonical donor splice site leading to exon 22 skipping (Fig. 2b). In order to assess these predictions and demonstrate the presence of the predicted out of frame transcripts lacking exon 22, we carried out RT-PCR experiments using total RNA extracted from the patient's blood cells. However, because of the low level of expression of PDE2A in blood cells and the possible instability of the out of frame transcript lacking exon 22, RTPCR was followed by a nested PCR using an internal couple of primers where the forward primer covers the $3^{\prime}$ end of exon 21 and the $5^{\prime}$ end of exon 23 and the reverse primer is located in exon 24 (Fig. 2c). This nested PCR is expected to specifically favors the amplification of the abnormal transcript. Sanger sequencing of the amplified fragment allowed to confirm exon 22 skipping (Fig. 2d) and therefore the
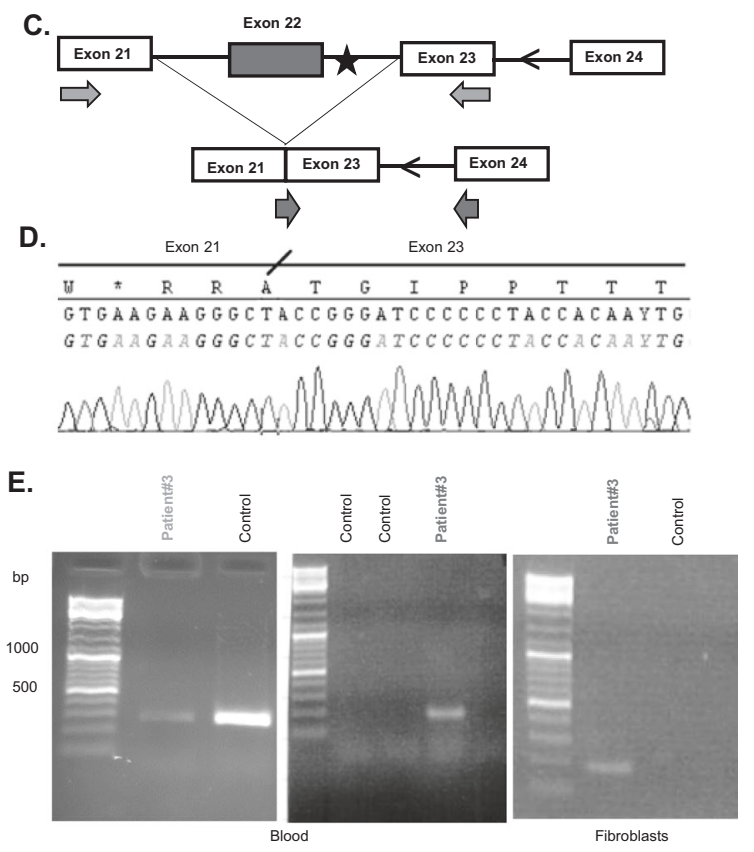

TTTGTGCCTCTGTG3') and nested PCR: Ex21-23F2:5'GCTGCC ATTGACTCCAATTT3'-Ex24R2:5'CCTCGAGGTAGTTGGTGAG $\mathrm{C}^{\prime}$ ) to evidence exon 22 skipping. d Sanger sequencing chromatograms of the amplified fragment confirming exon 22 skipping. e $1 \%$ gel electrophoresis initial RT-PCR products (left panel) and nested PCR products showing the amplification of the predicted fragment of about 184 bp only in the patient (blood: middle panel and fibroblast: right panel) bearing the variant and not in the control individual.

deleterious effect of the variant on PDE2A transcript splicing. As illustrated in Fig. 2e (middle panel), RT-PCR followed by nested PCR allowed to amplify the predicted fragment of about $184 \mathrm{bp}$ only in the patient bearing the variant and not in the control individual. These results were also confirmed through analyses of the expression of PDE2A transcripts using total RNA extracted from the patient's fibroblasts (Fig. 2e, right panel). Analysis of the mitochondria morphology in fibroblasts from Patient \#3 and \#1, in comparison to control cells, showed that the mitochondrial network in patient's fibroblasts appears abnormal with thicker and more irregular mitochondrial filaments, sometimes concentrating around the nucleus (Fig. 3a). The significant dysorganisation of the mitochondrial network in Patient \#3 and \#1cells (Fig. 3b) suggests an imbalance between mitochondria fusion and fission mechanisms due to the PDE2A variants.

\section{Discussion}

So far, only one patient with childhood-onset choreodystonia preceded by paroxysmal dystonia and associated 
Fig. 3 Mitochondrial morphology differs between control and affected cells. a Fibroblasts from non-affected (ctrl) individuals and from Patient \#3 and \#1 were grown on coverslips stained with Mitotracker ${ }^{\mathrm{TM}}$ Red CMXRos and DAPI. and observed by fluorescence microscopy. A mitochondrial phenotype corresponding to thicker, more irregular mitochondrial filaments was observed in Patient \#3 and \#1 cells. 9X zoom of the boxed region is displayed to allow a more detailed observation of the mitochondrial network. b Cells presenting abnormal mitochondrial network were counted in three independent experiments encompassing 100 cells each. The mean of the three experiments was calculated and significance determined using a $T$ test, $* * p<0.001$.
A mitotracker + DAPI
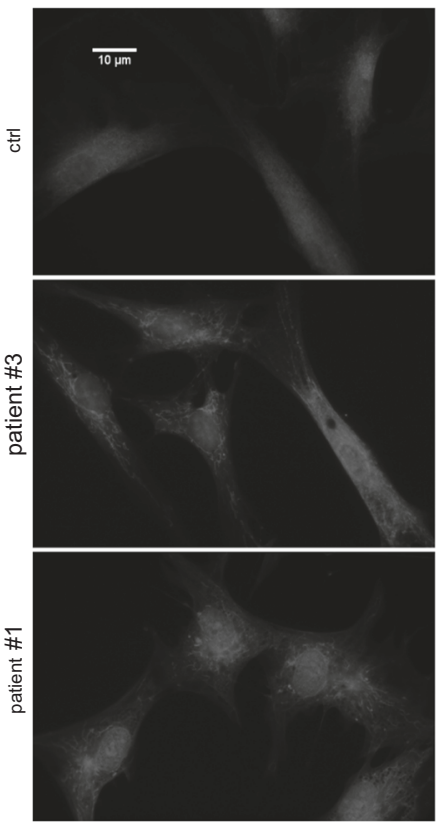

mitotracker
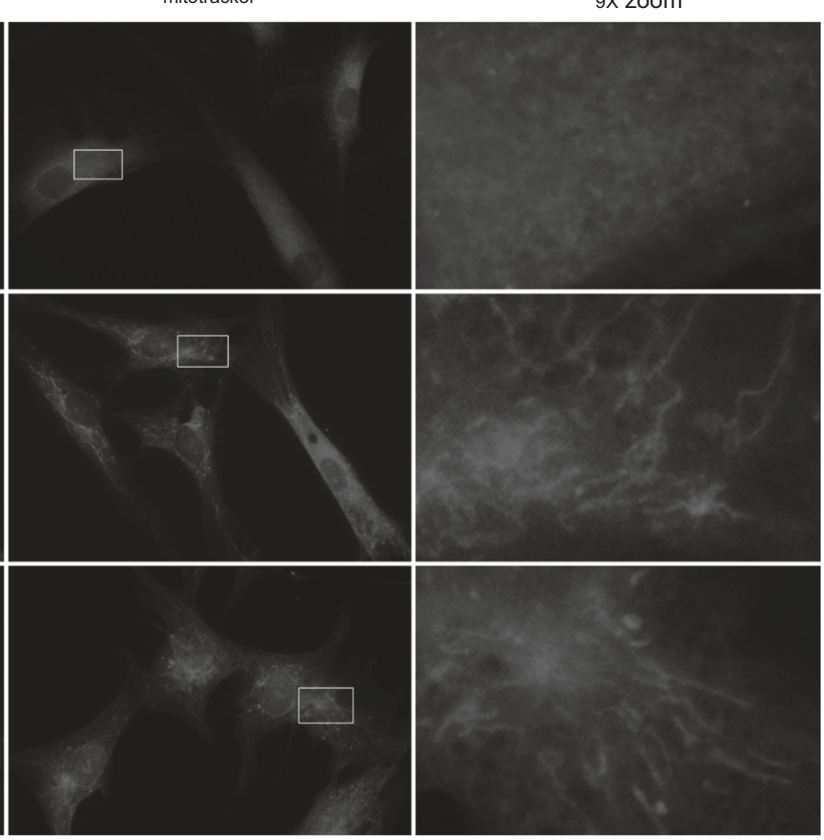

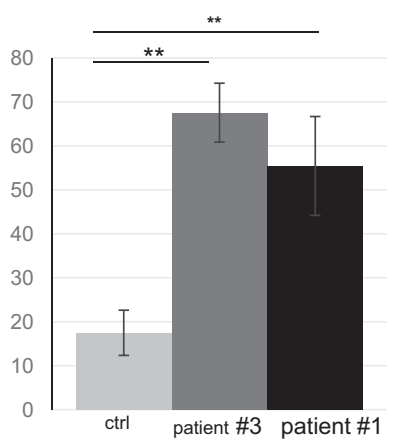

with cognitive impairment and interictal EEG abnormalities caused by homozygous missense variant in $P D E 2 A$ was reported. Here, we describe three new patients with biallelic $P D E 2 A$ variants confirming and strengthening that biallelic $P D E 2 A$ variants are involved in refractory paroxysmal dyskinesia with cognitive impairment, sometimes associated with choreodystonia and interictal baseline EEG abnormalities or epilepsy.

Along with the previously reported patient [8], all three patients reported to date had intellectual disability or developmental delay. However, clinical features of our Patient \#1 only are similar to those reported by Salpietro et al. [8] (Table 1). Like Patient \#1, this 12-year-old male patient first had frequent PD lasting few seconds at the age of 2 years (17 months in our case) and chronic choreic/ dystonic movements at 9 years ( 2 years in our case). Patient \#3, the oldest of all, also had PD but did not develop chronic choreodystonia, indicating that it is an inconstant feature of $P D E 2 A$-related disorder.
PDE2A-related PD is often misdiagnosed as epilepsy, which explains the delay of the diagnosis. However, absence of ictal EEG anomalies, usually rules out the hypothesis. The patient reported by Salpietro et al. had no epilepsy but interictal EEG abnormalities, like Patients \#1 and \#3 reported here. EEG recordings performed in Patient \#1 did not show ictal epileptic anomalies but their interpretation was extremely difficult due to movement artifacts. Thus, diagnosing nonepileptic paroxysms in patients with PDE2A-related PD may be challenging. Furthermore, Patient \#2, the youngest of all patients known to date, who had no PD to date, may be because of his young age, had a proven epilepsy wtih spasms and focal seizures (Fig. 4). This shows that the spectrum of the disease associated with $P D E 2 A$ variants includes epilepsy.

Moreover, detailed examination of clinical features of the three patients with PDE2A-related PD (Patients \#1 and \#3 and Salpietro et al.) allow to define the following characteristics of the condition: (i) dyskinesias start between the 


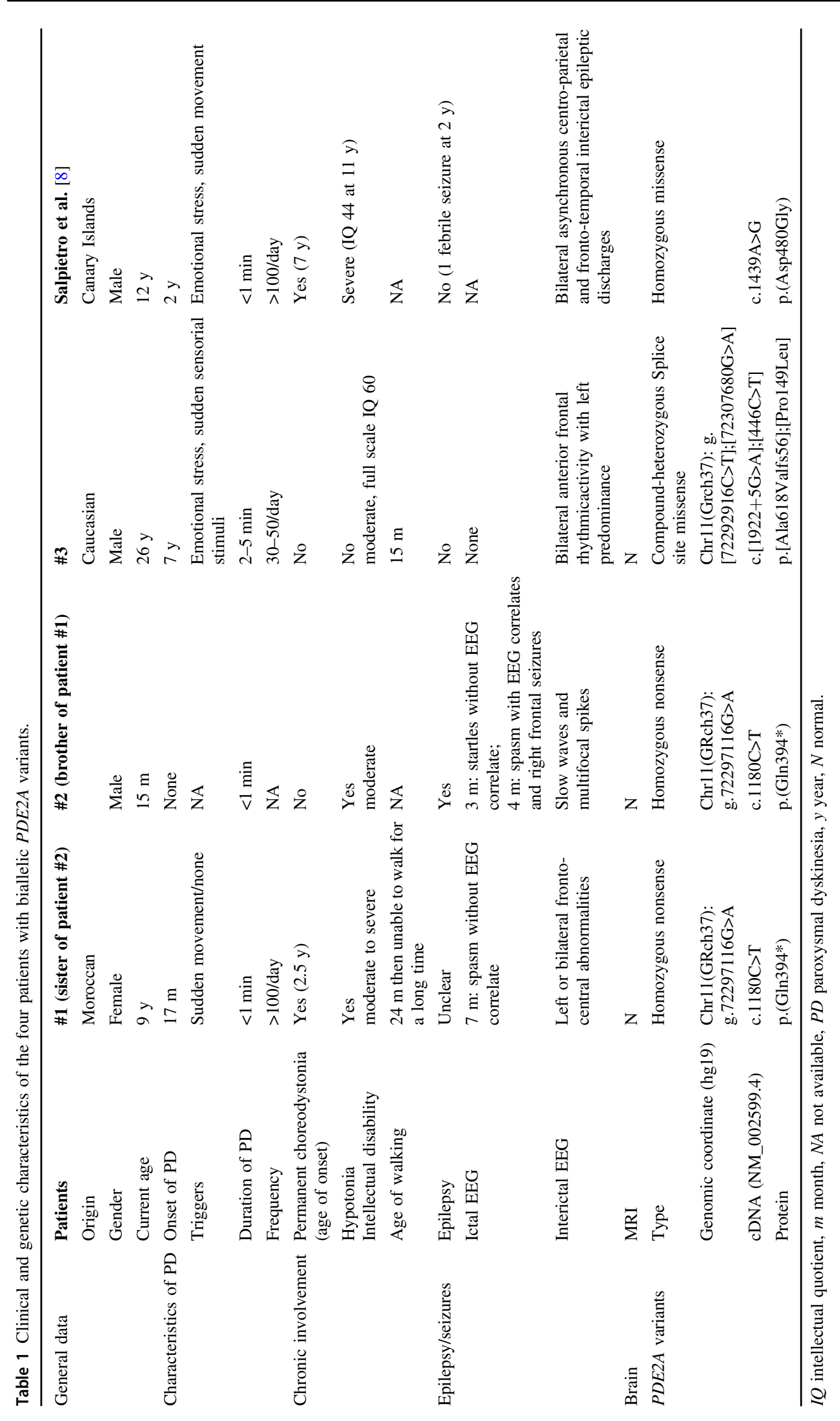


[SENS $* 10$ HF $* 120$ TC $* 0.3$ CAL $* 501$

A

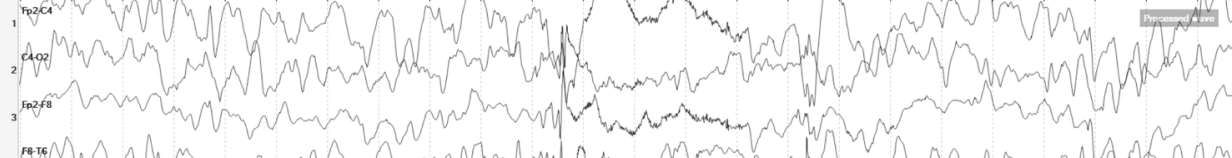
-

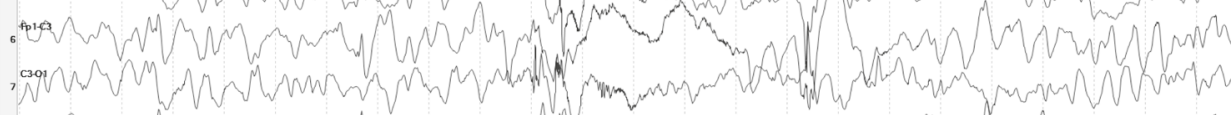

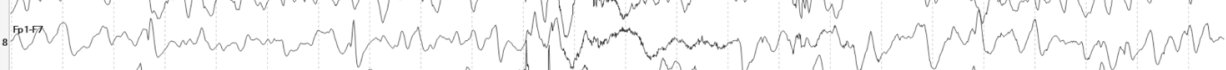

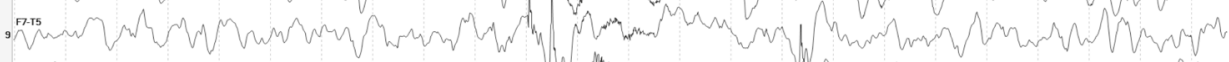

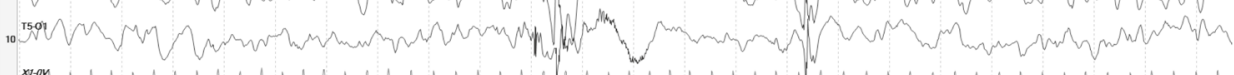

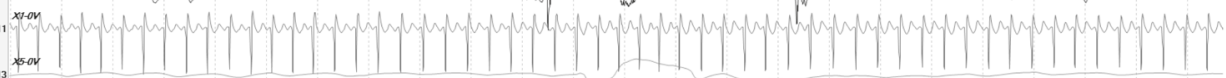
PGIfec Right deltoid

Left deltoid $\quad$ Sens $10 \mu \mathrm{N}$ CT $0,1 \mathrm{~s} \mathrm{HF} 35 \mathrm{~Hz}$

B

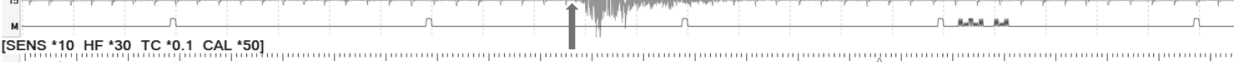

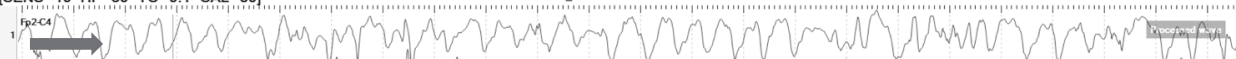

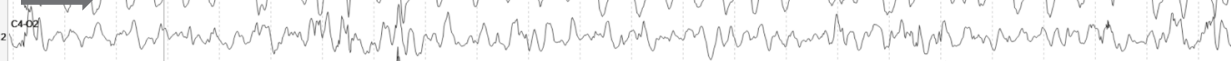

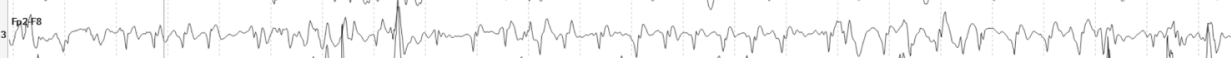

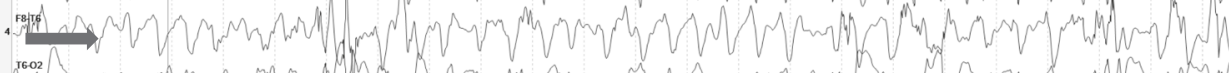

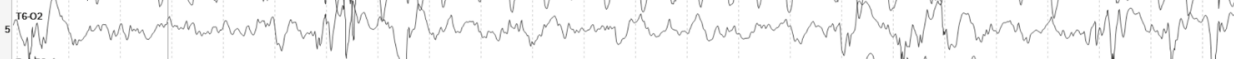

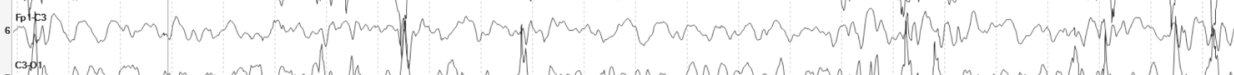

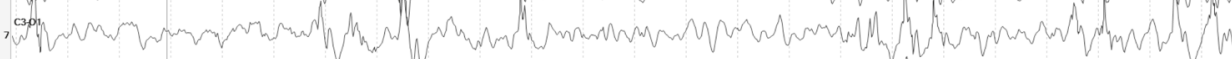
- int

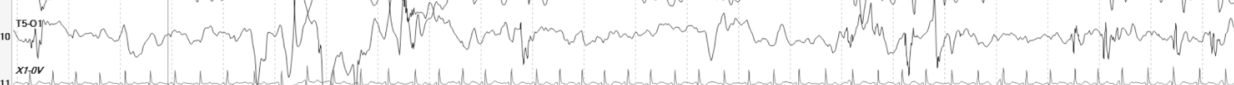

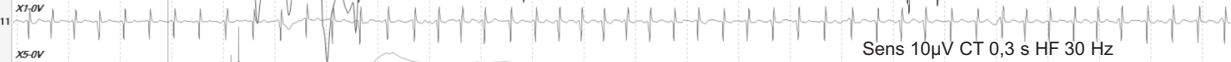
xoov Sens $10 \mu \mathrm{V} \mathrm{CT} 0,3 \mathrm{~s} \mathrm{HF} 30 \mathrm{~Hz}$

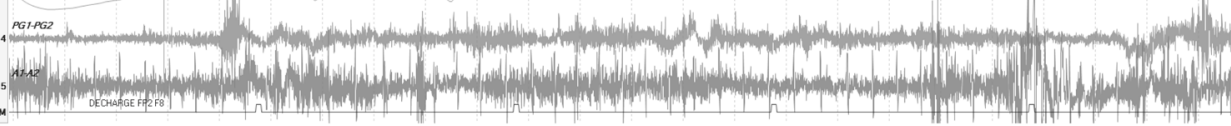

C

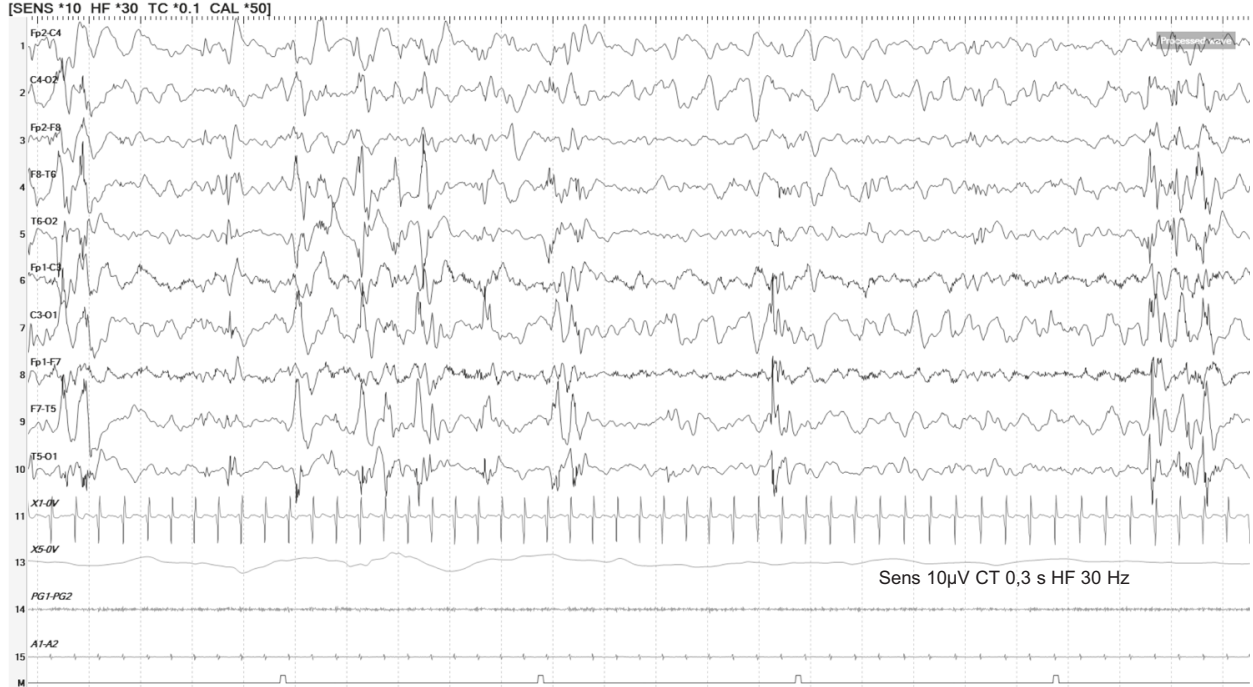

Fig. 4 Patient \#2 EEG recording at the age of 4 months. a Tonic symmetric contractions during awakening on EMG (arrow) with corresponding pattern of spasm on EEG. b Focal right seizure associating loss of contact, stretching of the arms, ocular revulsion, and left head deviation with rhythmic right discharge on EEG (arrow). c Intercritic awakening activity with multifocal epileptic pseudoperiodic spikes. 
ages of 17 months to 7 years, (ii) are frequent to very frequent ( 30 to $>100$ per day), (iii) last between $20 \mathrm{~s}$ to $5 \mathrm{~min}$, (iv) may be triggered by sudden movements, emotional stress, sudden sensorial stimuli, or appear to occur spontaneously, sometimes during sleep, (v) are refractory to medications, (vi) a diagnosis of epileptiform abnormalities does not exclude $P D E 2 A$ variants and (vii) patients have intellectual disability or developmental delay and (viii) interictal chronic choerodystonia may be present. Thus, $P D E 2 A$ variants may be considered as a cause of complex PKD, stimuli-induced PD and PNKD, as well as a cause of $P D$ with or without chronic choreodystonia.

PDE2A-related PD are pharmacoresistant. Chronic deep brain stimulation (DBS) of the internal globus pallidus has been reported to be beneficial in patients with dystonia refractory to medication used for movement disorders $[14,15]$. Nevertheless, DBS performed in the patient reported by Salpietro et al. showed improvement in the first weeks after chronic stimulation but followed by a very little improvement of PD without functional amelioration [16]. These data indicate that a better understanding of the $P D E 2 A$ variants consequences on basal ganglia and connected brain structures is needed to adapt DBS therapeutic approach to PDE2A-related disorders [17-19].

Phenotypic similarities such as truncal hypotonia and facial myokymias are observed in PDE2A-related PD as well as in PD due to $A D C Y 5$ heterozygous variations. The involvement of both PDE2A and ADCY5 in PD, together with variants in PDE10A underlying childhood-onset chorea [9-11], highlights the possible critical contribution of cyclic adenosine monophosphate (cAMP) and cyclic guanosine monophosphate (cGMP) metabolism and signaling in basal ganglia structures and circuitry to the pathophysiological mechanisms underlying hyperkinetic movement disorders. Phosphodiesterases are a superfamily of enzymes encoded by 21 genes that contain 11 isozymes according to the structural similarity [20]. They regulate the homeostasis of cAMP and cGMP which increases the strength of excitatory neural circuits and/or decreases inhibitory synaptic plasticity [21]. Each PDE gene has a specific expression pattern at the tissue and cellular levels [22]. Like other phosphodiesterases, $P D E 2 A$ is widely expressed in the human brain. It has a complex biochemical regulation and has the ability to hydrolyze both cAMP and cGMP, which are ubiquitous intracellular second messengers involved in numerous brain cellular functions, including neurotransmitter specification, axon guidance and refinement of neuronal connectivity [23-27]. The homozygous p. (Asp480Gly) PDE2A variant reported by Salpietro et al. affects the GAF-B domain of the $3^{\prime}, 5^{\prime}$ nucleotide phosphodiesterase PDE2A and severely decreases its enzymatic activity, which induces the accumulation of cAMP [8].
The homozygous nonsense variant (c.1180C $>\mathrm{T}$; p. $(\mathrm{Gln} 394 *))$ found in Patient \#1 and \#2, confirms that PED2A-related PD is due to loss-of-function. Likewise, the splice site variant c. $1922+5 \mathrm{G}>\mathrm{A}$ leading to exon 22 skipping and disruption of the reading frame found in Patient \#3 in association with the heterozygous missense p.(Pro149Leu) concerning a highly conserved amino acid of the protein sequence across species also suggest that these biallelic variants could lead to loss-of-function of PDE2A and accumulation of cAMP. Impaired cAMP and cGMP metabolism has also been demonstrated in two recent studies reporting dominant and recessive non-synonymous variants in PDE10A in hyperkinetic movement disorders $[28,29]$. Heterozygous variants in $A D C Y 5$, encoding the adenyl cyclase 5 enzyme that contribute to the synthesis of cAMP, are responsible for a large spectrum of infantile/ childhood-onset phenotypes that include nonprogressive choreiform movement disorders and PD [30, 31]. These variants induce the accumulation of cAMP due to gain-offunction [7]. Altogether, these data emphasize on the critical role of cAMP and cGMP regulation in basal ganglia functioning and pathophysiological mechanisms involved in several forms of hyperkinetic movement disorders.

One should also keep in mind that PDE2A is also expressed in the mitochondria and modulates cAMP and cGMP dependent signaling involved in the respiratory chain regulation [32]. PDE2A2 belongs to a cAMP/PKA signaling pathway located at the mitochondria and regulates PKA-dependent phosphorylation of the dynamin-related protein 1 (DRP1) [9]. The DRP1 GTPase is a key protein in mitochondrial fission [33]. Mitotracker ${ }^{\mathrm{TM}}$ Red staining shows that, in patient\#3 and \#1 fibroblast, the mitochondrial network was impaired with thicker and more irregular mitochondrial filaments (Fig. 3). This suggests an imbalance between mitochondria fusion/fission mechanisms linked to $P D E 2 A$ variation that could be linked to its phosphodiesterase enzymatic activity [34]. To our knowledge, it is the first report demonstrating that biallelic variants in $P D E 2 A$ alter the mitochondrial network organization and contribute in the pathogenesis of hyperkinetic movement disorders.

In conclusion, these findings together with those reported by Salpietro et al. indicate that biallelic variants in $P D E 2 A$ leading to loss of function are involved in heterogeneous phenotypes characterized by early-onset paroxysmal hyperkinetic movement disorders associated with cognitive impairment and possibly epilepsy. They also highlight the critical role of cAMP and cGMP metabolism in basal ganglia and in mitochondria functioning and homeostasis and their potential contribution in the pathophysiological mechanism underlying hyperkinetic movement disorders. 


\section{Online links}

https://databases.lovd.nl/shared/genes/PDE2A Individual ID 00295509 (Patient \#1); ID 00295764 (Patient \#2); ID 00264034 (Patient \#3)

ExAC database http://exac.broadinstitute.org/

Mutation Taster http://mutationtaster.org/

Gnomad database http://gnomad-old.broadinstitute.org/faq

ALAMUT Alamut Visual version 2.11 (Interactive

Biosoftware, Rouen, France)

Intergrative Genomics Viewer (IGV) version 2.4.7

The Human Gene Mutation Database http://www.hgmd. cf.ac.uk/ac/index.php

"Supplementary Information file is available at European journal of Human Genetics website"

Acknowledgements We would like to thank the patients and their parents for their participation in this study. This work was funded by CNRS, INSERM, Université de Strasbourg.

\section{Compliance with ethical standards}

Conflict of interest The authors declare that they have no conflict of interest.

Publisher's note Springer Nature remains neutral with regard to jurisdictional claims in published maps and institutional affiliations.

\section{References}

1. Bhatia KP. Paroxysmal dyskinesias. Mov Disord. 2011;26: 1157-65.

2. Gardiner AR, Jaffer F, Dale RC, Labrum R, Erro R, Meyer E, et al. The clinical and genetic heterogeneity of paroxysmal dyskinesias. Brain. 2015;138:3567-80.

3. McGuire S, Chanchani S, Khurana DS. Paroxysmal dyskinesias. Semin Pediatr Neurol. 2018;25:75-81.

4. Ebrahimi-Fakhari D, Saffari A, Westenberger A, Klein C. The evolving spectrum of PRRT2-associated paroxysmal diseases. Brain. 2015;138:3476-95.

5. Schneider SA, Paisan-Ruiz C, Garcia-Gorostiaga I, Quinn NP, Weber YC, Lerche $\mathrm{H}$, et al. GLUT1 gene mutations cause sporadic paroxysmal exercise-induced dyskinesias. Mov Disord. 2009;24:1684-8

6. Erro R, Bhatia KP, Espay AJ, Striano P. The epileptic and nonepileptic spectrum of paroxysmal dyskinesias: channelopathies, synaptopathies, and transportopathies: the pathophysiology of paroxysmal dyskinesias. Mov Disord. 2017;32:310-8.

7. Chen D-H, Méneret A, Friedman JR, Korvatscha O, Gad A, Bonkowski ES, et al. ADCY5-related dyskinesia: broader spectrum and genotype-phenotype correlations. Neurology. 2015;85:2026-35.

8. Salpietro V, Perez-Dueñas B, Nakashima K, San Antonio-Arce V, Manole A, Efthymiou S, et al. A homozygous loss-of-function mutation in $P D E 2 A$ associated to early-onset hereditary chorea: a homozygous $P D E 2 A$ mutation causing chorea. Mov Disord. 2018:33:482-8.

9. Monterisi S, Lobo MJ, Livie C, Castle C, Weinberger M, Baillie $\mathrm{G}$ et al. PDE2A2 regulates mitochondria morphology and apoptotic cell death via local modulation of cAMP/PKA signalling. eLife. 2017;6:1-20.
10. Roth S, Heintzmann R. Optical photon reassignment with increased axial resolution by structured illumination. Methods Appl Fluoresc. 2016;4:045005.

11. Kirk EP, Barlow-Stewart K, Selvanathan A, Josephi-Taylor S, Worgan L, Rajagopalan S, et al. Beyond the panel: preconception screening in consanguineous couples using the TruSight One "clinical exome". Genet Med. 2018. https://doi.org/10.1038/ s41436-018-0082-9.

12. Souirti Z, Landré E, Mellerio C, Devaux B, Chassoux F. Neural network underlying ictal pouting ("chapeau de gendarme") in frontal lobe epilepsy. Epilepsy Behav. 2014;37:249-57.

13. Exome Aggregation Consortium, Lek M, Karczewski KJ, Minikel EV, Samocha KE, Banks E, et al. Analysis of protein-coding genetic variation in 60,706 humans. Nature. 2016;536:285-91.

14. Coubes P, Roubertie A, Vayssiere N, Hemm S, Echenne B. Treatment of DYT1-generalised dystonia by stimulation of the internal globus pallidus. Lancet. 2000;355:2220-1.

15. van Coller R, Slabbert P, Vaidyanathan J, Schutte C. Successful treatment of disabling paroxysmal nonkinesigenic dyskinesia with deep brain stimulation of the globus pallidus internus. Stereotact Funct Neurosurg. 2014;92:388-92.

16. Candela S, Vanegas MI, Darling A, Ortigoza-Escobar JD, Alamar M, Muchart J, et al. Frameless robot-assisted pallidal deep brain stimulation surgery in pediatric patients with movement disorders: precision and short-term clinical results. J Neurosurg Pediatr. 2018;22:416-25.

17. Narayanan DL, Deshpande D, Das Bhowmik A, Varma DR, Dalal A. Familial choreoathetosis due to novel heterozygous mutation in PDE10A. Am J Med Genet A. 2018;176:146-50.

18. Carecchio M, Mencacci NE. Emerging monogenic complex hyperkinetic disorders. Curr Neurol Neurosci Rep. 2017;17:97107. https://doi.org/10.1007/s11910-017-0806-2.

19. Niccolini F, Mencacci NE, Yousaf T, Rabiner EA, Salpietro V, Pagano $G$ et al. PDE10A and ADCY5 mutations linked to molecular and microstructural basal ganglia pathology: PDE10A and ADCY5 Mutations Pathology. Mov Disord. 2018;33:196165. https://doi.org/10.1002/mds.27523.

20. Conti M, Beavo J. Biochemistry and physiology of cyclic nucleotide phosphodiesterases: essential components in cyclic nucleotide signaling. Annu Rev Biochem. 2007;76:481-511.

21. Bender AT, Beavo JA. Cyclic nucleotide phosphodiesterases: molecular regulation to clinical use. Pharmacol Rev. 2006;58:488-520.

22. Bingham J, Sudarsanam S, Srinivasan S. Profiling human phosphodiesterase genes and splice isoforms. Biochem Biophys Res Commun. 2006;350:25-32.

23. Bender AT, Beavo JA. Specific localized expression of cGMP PDEs in Purkinje neurons and macrophages. Neurochem Int. 2004;45:853-7.

24. Shelly M, Lim BK, Cancedda L, Heilshorn SC, Gao H, Poor MM. Local and long-range reciprocal regulation of cAMP and cGMP in axon/dendrite formation. Science. 2010;327:547-52.

25. Shen K, Cowan CW. Guidance molecules in synapse formation and plasticity. Cold Spring Harb Perspect Biol. 2010;2:a001842.

26. Averaimo $\mathrm{S}$, Nicol $\mathrm{X}$. Intermingled cAMP, cGMP and calcium spatiotemporal dynamics in developing neuronal circuits. Front Cell Neurosci. 2014;8:1-10. https://doi.org/10.3389/fncel.2014. 00376.

27. Akiyama H, Fukuda T, Tojima T, Nikolaev VO, Kamiguchi H. Cyclic nucleotide control of microtubule dynamics for axon guidance. J Neurosci. 2016;36:5636-49.

28. Diggle CP, Sukoff Rizzo SJ, Popiolek M, Hinttala R, Schülke JP, Kurian MA, et al. Biallelic mutations in PDE10A lead to loss of striatal PDE10A and a hyperkinetic movement disorder with onset in infancy. Am J Hum Genet. 2016;98:735-43. 
29. Mencacci NE, Kamsteeg E-J, Nakashima K, R'Bibo L, Lynch DS, Balint B, et al. De Novo mutations in PDE10A cause childhoodonset chorea with bilateral striatal lesions. Am J Hum Genet. 2016;98:763-71.

30. Chang FCF, Westenberger A, Dale RC, Smith M, Pall HS, PerezDuenas B, et al. Phenotypic insights into ADCY5-associated disease. Mov Disord J Mov Disord Soc. 2016;31:1033-40.

31. Friedman JR, Méneret A, Chen D-H, Trouillard O, Vidailhet M, Raskind WH, et al. ADCY5 mutation carriers display pleiotropic paroxysmal day and nighttime dyskinesias. Mov Disord J Mov Disord Soc. 2016;31:147-8.
32. Acin-Perez R, Russwurm M, Günnewig K, Gertz M, Zoidl G, Ramos L, et al. A phosphodiesterase $2 \mathrm{~A}$ isoform localized to mitochondria regulates respiration. J Biol Chem. 2011;286:30423-32.

33. Smirnova E, Shurland DL, Ryazantsev SN, van der Bliek AM. A human dynamin-related protein controls the distribution of mitochondria. J Cell Biol. 1998;143:351-8.

34. Huang $\mathrm{P}, \mathrm{Yu} \mathrm{T}$, Yoon Y. Mitochondrial clustering induced by overexpression of the mitochondrial fusion protein Mfn2 causes mitochondrial dysfunction and cell death. Eur J Cell Biol. 2007;86:289-302.

\section{Affiliations}

Diane Doummar $^{1,2} \cdot$ Christel Dentel $^{3} \cdot$ Romane Lyautey $^{4} \cdot$ Julia Metreau $^{5} \cdot$ Boris Keren $^{6} \cdot$ Nathalie Drouot $^{7,8}$. Ludivine Malherbe $^{4}$. Viviane Bouilleret ${ }^{9}$. Jérémie Courraud ${ }^{7,8} \cdot$ Maria Paola Valenti-Hirsch $^{10} \cdot$ Lorella Minotti $^{11}$. Blandine Dozieres-Puyravel ${ }^{12}$. Séverine Bär ${ }^{4} \cdot$ Julia Scholly $^{10}$ • Elise Schaefer ${ }^{13,14}$. Caroline Nava ${ }^{6}$.

Thomas Wirth $\mathbb{D}^{15}$. Hala Nasser ${ }^{16,17}$. Marie de Salins ${ }^{1}$. Anne de Saint Martin ${ }^{18}$. Marie Thérèse Abi Warde ${ }^{18}$. Philippe Kahane ${ }^{11}$ - Edouard Hirsch ${ }^{10}$ - Mathieu Anheim ${ }^{7,8,19}$. Sylvie Friant $\mathbb{D}^{4}$ - Jamel Chelly ${ }^{7,8,20}$. Cyril Mignot ${ }^{6,21,22} \cdot$ Gabrielle Rudolf $\mathbb{C}^{7,8,10,15}$

1 APHP, Service de Neuropédiatrie, Hôpital Armand Trousseau, Sorbonne Université, 75012 Paris, France

2 Centre de Référence Neurogénétique, Mouvement Anormaux de l'enfant, Hopital Armand Trousseau, 75012 Paris, France

3 Service de Neurologie, Centre Hospitalier Haguenau, Haguenau, France

4 Department of Molecular and Cellular Genetics, CNRS, GMGM, UMR7156, Université de Strasbourg, Strasbourg, France

5 APHP, Service de neurologie pédiatrique, Hôpital Universitaire Bicetre, Le Kremlin-Bicetre, France

6 APHP Département of Génétique, Hôpital Trousseau et Groupe Hospitalier Pitié-Salpêtrière, Sorbonne Université, Paris, France

7 Institut de Génétique et de Biologie Moléculaire et Cellulaire, CNRS U7104, INSERM U1258, 67400 Illkirch, France

8 Fédération de Médecine Translationnelle de Strasbourg (FMTS), Université de Strasbourg, Strasbourg, France

9 Service de neurophysiologie clinique et d'épileptologie, Hôpital Bicêtre, Assistance Publique des Hôpitaux de Paris, AP-HP, Le Kremlin-Bicêtre, France

10 Service d'Epileptologie, Hôpitaux Universitaires de Strasbourg, Strasbourg, France

11 GIN, Inserm U1216, Neurology Department Grenoble-Alpes university and hospital, Grenoble, France

12 Service de Neurologie Pédiatrique, AP-HP, Hôpital Robert Debré, 75019 Paris, France
13 Laboratoire de Génétique médicale, UMR_S INSERM U1112, IGMA, Faculté de Médecine FMTS, Université de Strasbourg, Strasbourg, France

14 Service de Génétique Médicale, IGMA, Hôpitaux Universitaires de Strasbourg, Strasbourg, France

15 Service des Pathologies du Mouvement, Hôpitaux Universitaires de Strasbourg, Strasbourg, France

16 Service des Explorations Fonctionnelles, Hôpital Robert Debré, 75019 Paris, France

17 Service de Génétique Clinique, Hôpital Robert Debré, 75019 Paris, France

18 Service de Neurologie Pédiatrique, Hôpitaux Universitaires de Strasbourg, Strasbourg, France

19 Service de Neurologie, Centre de Références des Maladies Neurogénétique Rares, Hôpitaux Universitaires de Strasbourg, Strasbourg, France

20 Service de Diagnostic Génétique, Hôpital Civil de Strasbourg, Hôpitaux Universitaires de Strasbourg, Strasbourg, France

21 Centre de Référence Déficiences Intellectuelles de Causes Rares, Strasbourg, France

22 INSERM, U 1127, CNRS UMR 7225, Sorbonne Université, UPMC Universié Paris 06 UMR S 1127, Institut du Cerveau et de la Moelle Epinière, ICM, Paris, France 